\title{
Identificación del Perfil de Carga en un Circuito Trifásico de Baja Tensión
}

\section{Identification of the Load Profile in a Three-Phase Low Voltage Circuit}

\author{
F. F. Obando, P. A. Bolaños, G. A. Palomino
}

Recibido: julio 22 de 2019 - Aceptado: enero 30 de 2021.

\begin{abstract}
${ }^{1}$ Resumen - La calidad de la red de potencia se ha ido convirtiendo en un aspecto muy importante para medir la calidad del servicio eléctrico, lo que implica mayor interés en conocer las características de la red eléctrica y disponer de información acerca de incidencias posibilita analizar los eventos, perturbaciones que resaltan en el sistema eléctrico previniendo los efectos que las provocan. El presente documento analiza criterios para la evaluación del perfil de carga mediante la identificación de las firmas de consumo, realizando el estudio de cargas conectadas en un ambiente controlado, con el fin de proponer un procedimiento para la identificación de las misma, verificando el comportamiento de dichas cargas en una red eléctrica.
\end{abstract}

Palabras clave - Calidad de energía, firmas de consumo, perfil de carga, procedimiento, red eléctrica, sistema de potencia.

Abstract-The quality of the power grid has become a very important aspect to measure the quality of the electric service, which implies greater interest in knowing the characteristics of the network, also having information about incidents makes it

${ }^{1}$ Producto derivado del proyecto de investigación "Evaluación del Perfil de Carga de un Circuito Eléctrico a partir de las Firmas de Consumo de una Carga Instalada". Grupo de investigación AI del departamento de electrónica instrumentación y control, Facultad de Ingeniería Electrónica y Telecomunicaciones de La Universidad del Cauca.

F.F. Obando, Universidad del Cauca, Popayán, Colombia, fobando@unicauca.edu.co

P.A. Bolaños, Universidad del Cauca, Popayán, Colombia, paubolanos@unicauca.edu.co

G.A. Palomino, Universidad del Cauca, Popayán, Colombia, alejapalom@unicauca.edu.co

Como citar este artículo: Obando, F. F., Bolaños P. A., Palomino, G. A. Identificación del Perfil de Carga en un Circuito Trifásico de Baja Tensión, Entre Ciencia e Ingeniería, vol. 15, no. 29, pp. , julio-diciembre 2020. DOI: https://doi.org/ 10.31908/19098367.2663.

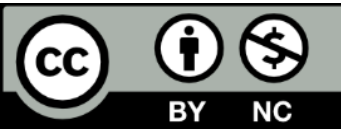

Attribution-NonCommercial 4.0 Intenational (CC By-NC 4.0) possible to analyze events, disturbances that stand out in the electrical system preventing the effects they cause. This article analyzes criteria for the evaluation of the load profile through the identification of the consumption signatures, carrying out the study of connected loads in a controlled environment, in order to propose a procedure for the identification of it, verifying the behavior of loads in an electrical grid.

Descriptors - Quality of energy, signatures of waveform, load profile, procedural, electrical grid, power system.

\section{INTRODUCCIÓN}

La calidad en la potencia eléctrica se ha convertido en un factor importante tanto para el operador de red, así como para el consumidor final; dada la naturaleza y especificaciones de las cargas conectadas a la red la presencia de perturbaciones y generación de armónicos hace que los equipos conectados estén sometidos a alteraciones por la propagación de señales diferentes a una onda sinusoidal propia [1]. Es así que, la calidad de tensión se ha ido convirtiendo en un aspecto relevante debido a la naturaleza de las cargas y su variación en el mercado eléctrico, lo que implica un mayor interés en conocer las características de las señales de la red y las perturbaciones en los parámetros de la onda eléctrica de sus valores nominales [1], entonces registrar y obtener un perfil de la carga conectada, es decir, conocer las características de la misma, en señales eléctricas suministradas junto con las perturbaciones que se producen en la red (hundimiento armónicos, sobretensiones, flicker, etc.), hace necesario reflejar estos fenómenos con indicadores de calidad de la continuidad del servicio para ser solucionadas e implementar las medidas adecuadas y evitar su propagación. Entonces, el hecho de que una incidencia vaya ligada a la actuación de un elemento de la red o maniobra permite relacionar episodios y eventos para determinar el origen de la perturbación y delimitar responsabilidades en caso necesario (pérdida de la producción por la parada de una línea de producción debido a 
un hundimiento) [23], discerniendo si la causa de la perturbación la tiene el operador de red o el cliente, debido al tipo de cargas conectadas. Así la vigilancia y seguimiento de la calidad de tensión, permite garantizar que las características de la energía suministrada cumplen las especificaciones fijadas en la legislación vigente y las acordadas entre las dos partes. Además, disponer de información acerca de incidencias y eventos, posibilita analizar las posibles causas de las perturbaciones en el sistema, así como los efectos que provocan [2].

Para conocer y controlar estas perturbaciones, su identificación sistemática es esencial y se plantea como un gran desafío. Además de los métodos primarios basados en la inspección visual de la forma de onda de la perturbación, los diferentes dispositivos de control, como los analizadores de perturbaciones y los analizadores de armónicos, se utilizan normalmente en la industria [24]. Estos analizadores generalmente emplean técnicas basadas en la comparación punto por punto de los valores rms de la señal distorsionada con su señal pura correspondiente, y / o la transformación de los datos en el dominio de la frecuencia a través de la transformada de Fourier (FT). Sin embargo, el principal problema de las herramientas de análisis tradicionales basadas en la transformada de Fourier es que no proporcionará suficiente información sobre el dominio del tiempo [20].

Esta situación ha hecho que se analicen los problemas más frecuentes en la señal de potencia, e identificar las firmas de cargas o perfil de cargas en tiempo real, como la distorsión armónica en las formas de onda de tensión y de corriente, ya que afectan la calidad de la potencia eléctrica, entonces así se podría definir los efectos en el funcionamiento de los equipos eléctricos. Por ejemplo, en los sistemas eléctricos de potencia existen señales de red industriales y comerciales representativas, en el caso de los motores de inducción su sensibilidad a los armónicos produce variaciones en la fuente de potencia, lo que se refleja en su rendimiento y características de operación [3].

Con respecto a Colombia, existe un crecimiento acelerado del consumo de energía eléctrica en múltiples sectores de la economía nacional [4]. Lo que indica, que este recurso es de vital importancia y particularmente es indispensable para el desarrollo de la industria, que es uno de los sectores con mayores índices de consumo energético a nivel nacional y también una de las principales causas de perturbación en la red, por las cargas usadas en el sector y las señales de la red eléctrica [5].

Ante la situación planteada, es necesario abordar el estudio del efecto en la calidad de la potencia por la variación de las cargas en tiempo real, para compensar esto es necesario saber qué tipo de cargas se conectan a la red y, también analizar la incidencia sobre otras cargas. El desarrollo de este procedimiento establece criterios y soportes obtenidos por correlación de datos, asignando un peso a los parámetros encontrados de las formas de onda de corriente y voltaje según su comportamiento, seguido a esto, con la implementación del sistema hardware - software y a la aplicación de dichos criterios, se logra evaluar el perfil de carga de un circuito eléctrico conectado asociándolo a la firma de consumo, por último, se realizaron pruebas de validación a cargas de tipo
$R L C, R C, R L$, Motor de inducción con rotor jaula de ardilla, Transformadores y cargas desconocidas, para comprobar los criterios establecidos por el procedimiento propuesto.

\section{FIRMAS DE CONSUMO DE LA RED ELÉCTRICA}

\section{A. Antecedentes}

El rendimiento de la red de potencia depende de cuatro componentes principales, las características de la línea, las características de la fuente, los condensadores de corrección del factor de potencia y, lo más importante, la carga [4]. Es por ello que la obtención y medición del evento presentado en la red de potencia incluyendo la carga, presenta particularidades que definen las firmas de consumo, dichas medidas incluyen: forma de onda de corriente en el dominio del tiempo, esta medida proporciona uno de los conjuntos de información más completos de la carga, ya que una alta resolución de la señal puede reflejar las características detalladas de un dispositivo, por otra parte, las potencias activa / reactiva $(P Q)$ son las mediciones más utilizadas para conocer el comportamiento de una carga, por lo que se pueden agrupar de acuerdo al consumo de potencia, factor de potencia o según la distorsión armónica generada [7], otras medidas importantes son, forma de onda de admitancia instantánea, forma de onda de potencia, valores propios y forma de onda transitoria de conmutación [5]. Es preciso resaltar que las características se pueden definir respecto a los datos que se puedan obtener en la medición del voltaje, corriente y potencia de la red, ya que los patrones de carga fluctúan y dependen de diversos factores que cambian dinámicamente [6].

De acuerdo con lo planteado en la literatura, para identificar varios dispositivos con diferentes características eléctricas, es beneficioso y necesario clasificarlos, es decir, construir una base de datos que contenga las firmas de carga medidas individualmente $\mathrm{y}$ poder agruparlas por características similares. Después, para lograr la desagregación de la carga es necesario plantear componentes claves que trabajen juntos para, adquirir información, analizar, tomar decisiones e implementar un software que contenga la solución, es decir, dividir el procedimiento de identificación en partes [8], por ejemplo:

Adquisición de datos: captura y almacenamiento de información crucial relacionada con individuos o grupos de cargas. Por supuesto, muchos datos de campo pueden ser monitoreados y registrados. Sin embargo, las aplicaciones pueden dictar el tipo de datos necesarios y las decisiones que deben tomarse [8].

Base de Datos: La base de datos debe incluir una amplia variedad de muestras de cargas, pero no es práctico recopilar las firmas de carga de todas las cargas diferentes, ya que hay una cantidad enorme de ellas [8].

Análisis: los métodos de análisis, obtención de conocimientos útiles y sugerencias de alternativas para obtener mejores resultados. Debido a la naturaleza estocástica de las cargas para sistemas grandes, ciertos análisis pueden requerir recursos computacionales intensivos y modelos analíticos sofisticados para tomar una carga compuesta y dividirla en un conjunto de firmas de carga de dispositivos identificables [8]. Analíticamente, un método de mayor uso es la correlación de 
datos. Es la forma numérica en la que la estadística ha podido evaluar la relación de dos o más variables. La cuál, entrega un coeficiente de correlación que es un valor cuantitativo de la relación entre dichas variables y puede variar desde $-1,00$ hasta $+1,00$. La correlación de proporcionalidad directa o positiva se establece con los valores $+1,00$ y de proporcionalidad inversa o negativa, con -1,00. No existe relación entre las variables cuando el coeficiente es de 0,00 [9].

Interfaz: La presentación de datos de carga, resultados de análisis, alternativas de control ya sean gráficos o analíticos son una parte fundamental del sistema para mostrar los resultados obtenidos [8].

\section{B. Desarrollo del Procedimiento Propuesto}

Se propone un procedimiento que permite identificar un tipo de carga instalada en una red eléctrica trifásica de baja tensión, para lo cual se definen las características eléctricas del circuito en estudio, es decir, niveles de tensión, configuración de conexión del circuito, ésta puede ser en delta o estrella, es necesario la verificación del escenario dado que debe ser un ambiente controlado, es decir, deben estar conectadas sólo las cargas de estudio sin dar lugar a perturbaciones que pueden alterar los valores de las mediciones realizadas. Posteriormente, se determina si la conexión es monofásica con una línea y neutro o trifásica y neutro, para luego realizar la ubicación del dispositivo de adquisición de datos y obtener las señales de voltaje y corriente. De acuerdo con estudios en la literatura, se realizaron mediciones de un sistema trifásico en las dos conexiones, delta y estrella, obteniendo las formas de onda de voltaje y corriente para una sola fase, con una frecuencia de $60 \mathrm{~Hz}$.

Es recomendable para el estudio tomar 12 ciclos de la señal y un tiempo de muestro de $20 \mu \mathrm{s}$ cada $200 \mathrm{~ms}$ respecto a lo expuesto en [13], [16], [18]-[19]. Una vez extraídas las formas de onda de voltaje y corriente se calculan parámetros como: potencia activa, reactiva y aparente, factor de potencia, distorsión total armónica de corriente y voltaje, forma o distorsión de onda, valores rms tanto de corriente y voltaje, armónicos impares de la señal, y factor de cresta, dichos parámetros son analizados aplicando la correlación como se indican en la Tabla III, es recomendable calcular la varianza y desviación estándar, identificando el nivel de dispersión y la variabilidad de los parámetros, lo que permite identificar cuáles tienen mayor presencia en la señal con menos cambios en su valor, la Tabla III contiene las expresiones para calcular dichos métodos estadísticos. Con este análisis, se encontró que para realizar el cálculo de los parámetros de identificación se deben medir mínimo 10 muestras de cada carga según los convertidores usados se estudian en [21] para aplicar el procedimiento, debido a que los datos probabilistas deben tener muestras considerables para que los resultados sean confiables. Después, se calcula el promedio o media aritmética para determinar el peso de cada parámetro en la señal, es decir, cuánto equivale en valor porcentual los datos que definen la carga, como el valor de los parámetros varía de acuerdo a las características de la carga se obtienen diferentes pesos para dichos parámetros en las diferentes cargas conectadas, definiendo así el perfil de carga. Entonces, las cargas que se estudiaron son, motor rotor jaula de ardilla, circuitos $R L C$ - RL-RC y trasformador, dichas cargas son conectadas como puede verse en la Fig. 1 y se explicara detalladamente cada una en el apartado $C$.

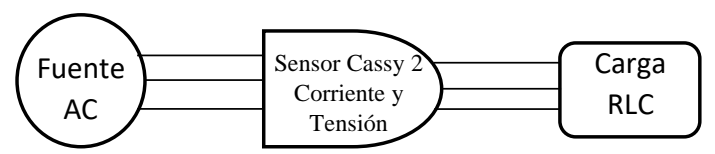

Fig.1. Conexión genérica de las cargas trifásica.

Finalmente, se calcula el error muestral con los parámetros de mayor representación para definir rangos de pertenencia de las cargas y otorgar una etiqueta o tag. La tabla III, presenta los parámetros a calcular con sus respectivas expresiones y en la Tabla IV se evidencian los pesos definitivos organizados de mayor a menor para cada parámetro en las cargas de estudio, estos pesos fueron calculados de acuerdo al promedio obtenido para cada uno, por ejemplo, los datos obtenidos de la toma de las 10 muestras para el motor tipo jaula de ardilla conectado a una red trifásica de baja tensión se indican en la tabla I

TABLA I

\begin{tabular}{|c|c|c|c|c|c|c|c|c|c|c|}
\hline \multicolumn{10}{|c|}{ MUESTREO PARA EL MOTOR JAULA DE ARDILLA } \\
\hline Dato & $\mathbf{1}$ & $\mathbf{2}$ & $\mathbf{3}$ & $\mathbf{4}$ & $\mathbf{5}$ & $\mathbf{6}$ & $\mathbf{7}$ & $\mathbf{8}$ & $\mathbf{9}$ & $\mathbf{1 0}$ \\
\hline FP & 0,60 & 0,60 & 0,60 & 0,60 & 0,60 & 0,60 & 0,60 & 0,60 & 0,60 & 0,60 \\
\hline THD_I & 0,01 & 0,01 & 0,01 & 0,01 & 0,01 & 0,01 & 0,01 & 0,01 & 0,01 & 0,01 \\
\hline RMS_I & 1,16 & 1,16 & 1,15 & 1,16 & 1,15 & 1,15 & 1,15 & 1,16 & 1,15 & 1,16 \\
\hline FC_I & 1,40 & 1,41 & 1,41 & 1,41 & 1,40 & 1,40 & 1,40 & 1,40 & 1,40 & 1,40 \\
\hline ARM_3_I & 0,01 & 0,01 & 0,01 & 0,01 & 0,01 & 0,00 & 0,01 & 0,01 & 0,00 & 0,00 \\
\hline ARM_5_I & 0,01 & 0,01 & 0,01 & 0,01 & 0,01 & 0,01 & 0,02 & 0,01 & 0,01 & 0,01 \\
\hline ARM_7_I & 0,00 & 0,00 & 0,00 & 0,00 & 0,00 & 0,00 & 0,00 & 0,00 & 0,00 & 0,00 \\
\hline THD_V & 0,02 & 0,02 & 0,02 & 0,02 & 0,02 & 0,02 & 0,02 & 0,02 & 0,02 & 0,02 \\
\hline FC_V & 1,45 & 1,45 & 1,45 & 1,45 & 1,45 & 1,45 & 1,45 & 1,44 & 1,44 & 1,45 \\
\hline ARM_3_V & 0,37 & 0,39 & 0,41 & 0,36 & 0,33 & 0,76 & 0,30 & 0,42 & 0,71 & 0,66 \\
\hline ARM_5_V & 1,88 & 1,69 & 1,82 & 1,85 & 1,75 & 1,76 & 2,02 & 1,99 & 1,74 & 2,03 \\
\hline ARM_7_V & 0,92 & 1,10 & 0,85 & 0,97 & 1,10 & 1,16 & 0,77 & 0,88 & 0,92 & 0,81 \\
\hline
\end{tabular}

Ahora bien, se promedian los valores de cada uno de los parámetros y se suman obteniendo así la media aritmética como se indica en la tabla II, que equivale a; Media $=7.9300$ la cual representa el $100 \%$ del peso total de esta carga.

Entonces como son 12 parámetros encontrados de acuerdo con su valor se definirá el peso de representación, en este caso el factor de potencia es igual a 0.6 entonces, sí 7.93 es el $100 \% 0.6$ sería X \%, es decir, se divide el 0.6 entre el Valor de la Media y se multiplica por $100 \%$ obteniendo el valor porcentual de cada parámetro, con la expresión (1) se puede encontrar el peso para cualquier parámetro.

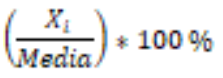

Así:

$\left(\frac{0,6}{7,930}\right) \approx 10096 \cong 7,5 \cong 796$ 
TABLA II

PROMEDIO DE PARÁMETROS Y OBTENCIÓN DE LA MEDIA ARITMÉTICA

\begin{tabular}{|c|c|}
\hline Dato & Prom \\
\hline FP & 0,6000 \\
\hline THD_I & 0,0131 \\
\hline RMS_I & 1,1542 \\
\hline FC_I & 1,4030 \\
\hline ARM_3_I & 0,0054 \\
\hline ARM_5_I & 0,0137 \\
\hline ARM_7_I & 0,0014 \\
\hline THD_V & 0,0199 \\
\hline FC_V & 1,4477 \\
\hline ARM_3_V & 0,4703 \\
\hline ARM_5_V & 1,8543 \\
\hline ARM_7_V & 0,9464 \\
\hline SUMA DEL PROMEDIO & $\mathbf{7 , 9 3 0}$ \\
\hline
\end{tabular}

TABLA III

PARÁMETROS DE LA CARGA

\begin{tabular}{|c|c|c|}
\hline Símbolo & Definición & Ecuación \\
\hline $\mathrm{P}$ & Potencia activa & $P=\sqrt{3} * V * I * \cos (q)$ \\
\hline Q & Potencia reactiva. & 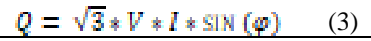 \\
\hline $\mathrm{S}$ & Potencia aparente & $S=\sqrt{P^{2}+Q^{2}}$ \\
\hline FP & Factor de Potencia & $P=\frac{\bar{Q}}{8}$ \\
\hline THDI & \begin{tabular}{|c|}
$\begin{array}{c}\text { Distorsión total armónica } \\
\text { de corriente }\end{array}$ \\
\end{tabular} & 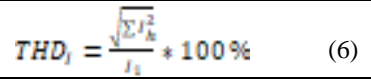 \\
\hline $\mathrm{VP}$ & Valor pico de voltaje & $V_{\mathrm{Hitsg}}=V_{r+m s} * 2 \sqrt{2}$ \\
\hline THDV & $\begin{array}{c}\text { Distorsión total armónica } \\
\text { de voltaje }\end{array}$ & $T H D_{V}=\frac{\sqrt{\operatorname{sig}^{2}}}{\sqrt{\mathbb{V}_{0}}} * 100 \%$ \\
\hline $\mathrm{CFI}$ & $\begin{array}{l}\text { Factor de cresta de } \\
\text { corriente. }\end{array}$ & $C F I=\frac{P_{\min }}{D_{\min }}$ \\
\hline $\mathrm{CFV}$ & $\begin{array}{l}\text { Factor de cresta de } \\
\text { voltaje. }\end{array}$ & $C F V=\frac{V_{\min }}{W_{\operatorname{mix}}}$ \\
\hline ARM_I & $\begin{array}{c}\text { Armónicos de corriente } \\
\text { Impares [5]. }\end{array}$ & 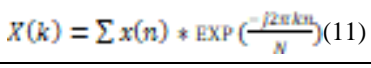 \\
\hline DF & $\begin{array}{c}\text { Forma o factor de } \\
\text { distorsión de corriente. }\end{array}$ & $D F=\frac{d_{0}}{I_{\max }}$ \\
\hline RMS & $\begin{array}{c}\text { Valor } r m s \text { de corriente } \\
\text { y voltaje. }\end{array}$ & 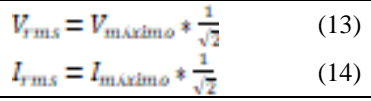 \\
\hline ARM_V & $\begin{array}{l}\text { Armónicos de voltaje } \\
\text { Impares [5] }\end{array}$ & (11) \\
\hline COEF & $\begin{array}{l}\text { Coeficiente de } \\
\text { correlación. }\end{array}$ & 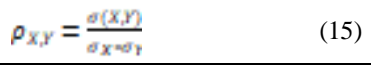 \\
\hline VARIANZA & Varianza. & $\alpha=\frac{1}{n} \sum_{i=1}^{n}\left(x_{i}-\vec{x}\right)^{2}$ \\
\hline Desvet & Desviación estándar. & 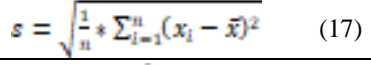 \\
\hline Error_M & Error muestral. & 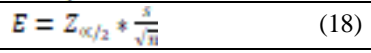 \\
\hline Promedio & $\begin{array}{l}\text { Promedio o Media } \\
\text { aritmética. }\end{array}$ & Promedio $=\frac{\mathrm{X}_{\mathrm{X}}}{\mathrm{R}}$ \\
\hline
\end{tabular}

TABLA IV

PESOS DEFINITIVOS DE LAS CARGAS

\begin{tabular}{|c|c|c|c|c|c|c|c|}
\hline Dato & $\begin{array}{c}\mathbf{M} \\
\mathbf{\%}\end{array}$ & $\begin{array}{c}\text { RC } \\
\mathbf{\%}\end{array}$ & $\begin{array}{c}\text { RL } \\
\boldsymbol{\%}\end{array}$ & $\begin{array}{c}\text { RLC } \\
\boldsymbol{\%}\end{array}$ & $\begin{array}{c}\text { Trafo } \\
\mathbf{\%}\end{array}$ & $\begin{array}{c}\text { Trafo. } \\
\text { Redu } \\
\mathbf{\%}\end{array}$ & $\begin{array}{c}\text { Trafo. } \\
\text { Eleva } \\
\mathbf{\%}\end{array}$ \\
\hline AR_5V & 23.0 & 30.0 & 26.0 & 24.0 & 26.0 & 28.0 & 26.0 \\
\hline FCV & 18.0 & 22.0 & 21.0 & 18.0 & 20.0 & 21.0 & 19.0 \\
\hline FCI & 17.0 & 22.0 & 20.0 & 17.0 & 19.0 & 20.0 & 19.0 \\
\hline RMS_I & 14.0 & 1.0 & 2.0 & 13.0 & 3.0 & 1.0 & 6.0 \\
\hline AR_7V & 12.0 & 12.0 & 13.0 & 11.0 & 11.0 & 10.0 & 11.0 \\
\hline FP & 7.0 & 4.0 & 11.0 & 10.0 & 12.0 & 13.0 & 12.0 \\
\hline AR_3V & 6.0 & 6.0 & 5.0 & 4.0 & 6.0 & 5.0 & 5.0 \\
\hline THD_V & 1.0 & 1.0 & 1.0 & 1.0 & 1.0 & 0.8 & 0.8 \\
\hline AR_5I & 0.7 & 0.5 & 0.5 & 0.8 & 0.7 & 0.2 & 0.2 \\
\hline THD_I & 0.7 & 1.0 & 0.3 & 1.0 & 1.0 & 0.8 & 0.8 \\
\hline AR_3I & 0.3 & 0.3 & 0.1 & 0.1 & 0.2 & 0.1 & 0.1 \\
\hline AR_7I & 0.3 & 0.2 & 0.1 & 0.1 & 0.1 & 0.1 & 0.1 \\
\hline
\end{tabular}

TABLA V

RANGOS DEFINITIVOS DE LAS CARGAS

\begin{tabular}{|c|c|c|c|c|c|c|c|c|c|c|c|}
\hline 宝 & $\overrightarrow{\mid l}$ & $\vec{n}_{\underline{n}}^{n}$ & $\underbrace{\prime \prime}$ & 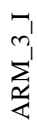 & $\sum_{\substack{c \\
4}}^{n}$ & $\frac{\sqrt{\prime}}{\sum^{\prime}}$ & $\begin{array}{l}> \\
\Theta^{\prime} \\
\text { 量 }\end{array}$ & $\underbrace{\prime}_{\text {U }}$ & $\begin{array}{l}> \\
m_{1}^{\prime} \\
\sum_{i}^{\prime}\end{array}$ & $\begin{array}{l}> \\
n \\
n_{1}^{\prime} \\
\sum_{4}\end{array}$ & 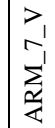 \\
\hline
\end{tabular}

\begin{tabular}{ll|l|l|l|l|l|l|l|l|l|}
\hline & & & & & & & & \\
\hline
\end{tabular}

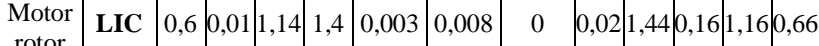

rotor

jaula de

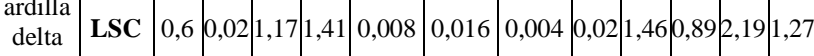

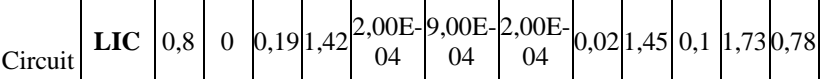

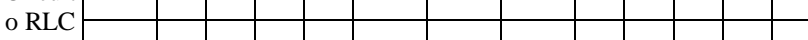

\begin{tabular}{llll|l|l|c|c|c|c|c|c|c|} 
delta & LSC & 0,8 & 0,01 & 0,2 & 1,42 & $5,00 \mathrm{E}-$ & $9,00 \mathrm{E}-3,00 \mathrm{E}-$ & 0,02 & 1,45 & 0,55 & 2,05 & 1,02 \\
0
\end{tabular}

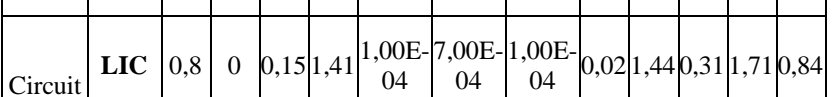

RL

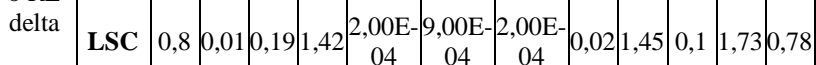

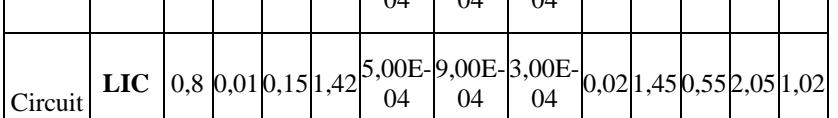

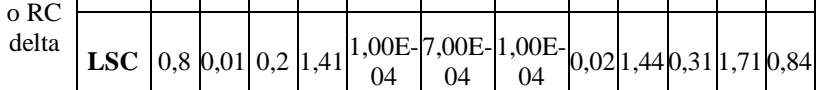

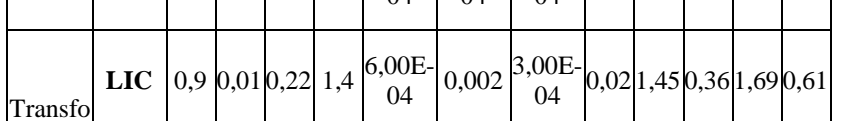

Transfo

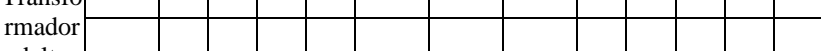

\begin{tabular}{lll|l|l|l|l|l|l|l|l|l|l|l|l} 
delta & LSC & 0,9 & 0,02 & 0,22 & 1,43 & 0,001 & 0,003 & $4,00 \mathrm{E}-$ & 0,02 & 1,45 & 0,6 & 2,19 & 0,98
\end{tabular}

\begin{tabular}{|c|c|c|c|c|c|c|c|c|c|c|c|c|c|}
\hline \multirow{2}{*}{$\begin{array}{l}\text { Transfo } \\
\text { rmador } \\
\text { reducto } \\
\text { r delta }\end{array}$} & LIC & 0,9 & 0,02 & 0,08 & 1,38 & $\begin{array}{c}9,00 \mathrm{E}- \\
04\end{array}$ & 0,002 & $\begin{array}{c}5,00 \mathrm{E}- \\
04\end{array}$ & 0,02 & 1,44 & 0,28 & 1,55 & 0,63 \\
\hline & LSC & 0,9 & 0,03 & 0,08 & 1,44 & 0,001 & 0,002 & $\begin{array}{c}5,00 \mathrm{E}- \\
04\end{array}$ & 0,02 & 1,45 & 0,49 & 2,14 & 0,91 \\
\hline \multirow{2}{*}{$\begin{array}{l}\text { Transfo } \\
\text { rmador } \\
\text { elevado } \\
\text { r delta }\end{array}$} & LIC & 0,9 & 0,01 & 0,46 & 1,39 & 0,001 & 0,003 & 0,002 & 0,02 & 1,45 & 0,31 & 1,87 & 0,71 \\
\hline & LSC & 0,9 & 0,01 & 0,49 & 1,41 & 0,002 & 0,003 & 0,002 & 0,02 & 1,4 & 0,47 & 2,11 & 1,02 \\
\hline
\end{tabular}


De esta forma, se calcula el peso para cada parámetro obtenido y se otorga un valor completo para asignar el peso total, también los rangos obtenidos son indicados en la tabla $\mathrm{V}$ para las cargas en conexión delta, estos datos se obtienen de las 10 muestras medidas para cada parámetro y se calcula el valor máximo y mínimo que puede tomar cada uno caracterizando las cargas. Se debe tener en cuenta el error muestral y se calcula con la expresión (18) de la tabla III, para este procedimiento dicho valor equivale a $1 \%$ de error que presenta el dispositivo de medición, luego es sumado para límite superior y restado para el límite inferior del parámetro definiendo los límites de comparación, igualmente en la tabla VI se observan las etiquetas de identificación asignadas para las diferentes cargas y por último, en la tabla VII se indica la plantilla que se elaboró para la identificación del motor jaula de ardilla conectado en configuración delta.

TABLA VI

ETIQUETAS O TAG DE IDENTIFICACIÓN

\begin{tabular}{|c|c|c|}
\hline \multicolumn{3}{|c|}{ ID PARA CADA CARGA } \\
\hline Carga & $\begin{array}{c}\text { Configuración } \\
\text { Delta }\end{array}$ & $\begin{array}{c}\text { Configuración } \\
\text { Estrella }\end{array}$ \\
\hline Motor & MIA_101 & MIA_102 \\
\hline Circuito RLC & RLC_101 & RLC_102 \\
\hline Circuito RL & RL_101 & RL_102 \\
\hline Circuito RC & RC_101 & RC_102 \\
\hline Transformador & T_101 & T_102 \\
\hline Transformador elevador & TE_101 & TE_102 \\
\hline Transformador reductor & TR_101 & TR_102 \\
\hline
\end{tabular}

TABLA VII

PLANTILLA DE IDENTIFICACIÓN PARA EL MOTOR JAULA DE ARDILLA

\begin{tabular}{|l|c|c|c|}
\hline \multicolumn{4}{|c|}{ Motor jaula de ardilla } \\
\hline CONEXIÓN: & \multicolumn{1}{l|}{ Delta } \\
\hline ID: & MJA_101 \\
\hline \multicolumn{1}{|c|}{ Parámetro } & Peso & $\begin{array}{c}\text { Rango } \\
\text { inferior }\end{array}$ & $\begin{array}{c}\text { Rango } \\
\text { superior }\end{array}$ \\
\hline FP & $7,58 \%$ & 0,6 & 0,6 \\
\hline THD_I & $0,16 \%$ & 0,01 & 0,0154 \\
\hline RMS_I & $14,57 \%$ & 1,1421 & 1,166 \\
\hline FACTOR_CRESTA_I & $17,72 \%$ & 1,395 & 1,4127 \\
\hline ARM_3_I & $0,07 \%$ & 0,003 & 0,0081 \\
\hline ARM_5_I & $0,17 \%$ & 0,0081 & 0,0164 \\
\hline ARM_7_I & $0,02 \%$ & $-0,0001$ & 0,0035 \\
\hline THD_V & $0,25 \%$ & 0,0166 & 0,0218 \\
\hline FACTOR_CRESTA_V & $18,29 \%$ & 1,4345 & 1,4567 \\
\hline ARM_3_V & $6,25 \%$ & 0,1607 & 0,89 \\
\hline ARM_5_V & $22,95 \%$ & 1,1554 & 2,1926 \\
\hline ARM_7_V & $11,97 \%$ & 0,6618 & 1,2679 \\
\hline
\end{tabular}

Por último, se realiza una validación realizando la obtención de las formas de onda de voltaje y corriente, verificando si la asignación de los pesos y etiquetas son adecuadas para la identificación de la carga o por el contrario, al realizar cambios provocados por desbalances en el circuito, la identificación no es posible, comprobando la sensibilidad del procedimiento. La Fig. 2 muestra el desarrollo del procedimiento desarrollado de forma general guiando paso a paso la aplicación del procedimiento.

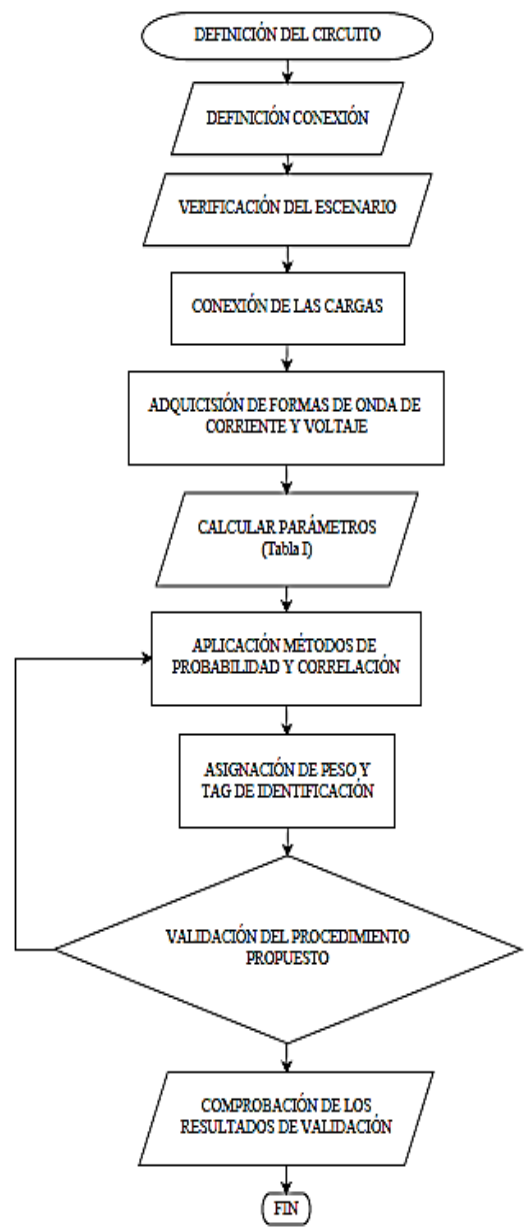

Fig.2. Diagrama de flujo del procedimiento propuesto.

\section{Resultados y Validación del Procedimiento Propuesto}

El sistema de adquisición de datos consta principalmente del sensor Cassy 2 (524 - 013) conectado por cable USB a un ordenador personal con su respectivo software Cassy lab 2 (Fig. 3) que permite guardar los datos en un archivo txt en la computadora, para fines de cálculo y tratamiento de señales.

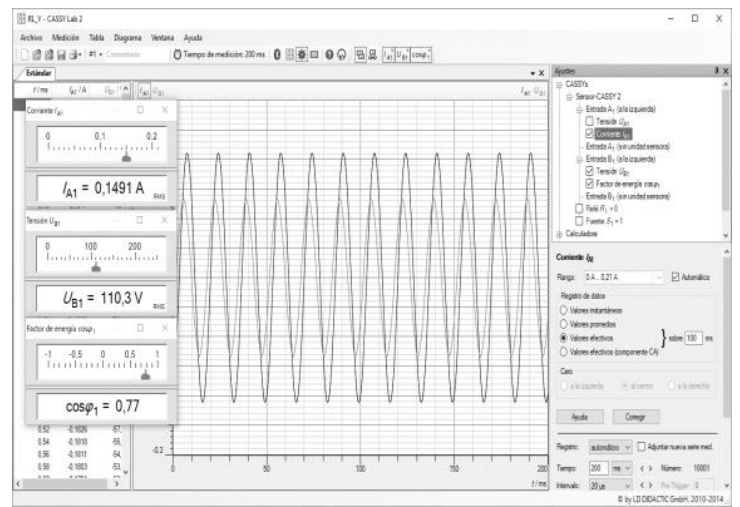

Fig. 3. Interfaz gráfica del software Cassy lab 2.

Las muestras tomadas en la entrada analógica de voltaje están limitadas a los siguientes datos técnicos:

- Cuenta con una resolución de 12 bits. 
- Su rango de medición va desde $\pm 0.1 \mathrm{~V}$ hasta $\pm 250 \mathrm{~V}$ y $\pm 0.03 \mathrm{~A}$ hasta $\pm 3 \mathrm{~A}$.

- Cuenta con una velocidad de escaneo de $1 \mathrm{MHz}$ por entrada, entonces se tiene una cantidad de 10.000 valores $/ \mathrm{s}$ hasta 20.000 valores $/ \mathrm{s}$.

Para la aplicación del método se tomaron en total 7 cargas de la planta lab_volt EMS 8341-2, con conexión en configuración delta o estrella, mencionadas en la tabla VIII.

TABLA VIII

CARGAS TOMADAS PARA EL PROCEDIMIENTO

\begin{tabular}{|l|l|}
\hline \multicolumn{1}{|c|}{ Carga } & \multicolumn{1}{c|}{ Características } \\
\hline Circuito RLC & $\begin{array}{l}\mathrm{R}=300 \Omega, \mathrm{L}=3.2 \mathrm{H}, \mathrm{C}=8.8 \mu \mathrm{F}, \\
252 \mathrm{VAR}-120 \mathrm{~V}, 60 \mathrm{~Hz}, \text { precisión de } \pm 5 \%, \mathrm{Q}=10 .\end{array}$ \\
\hline Circuito RL & $\begin{array}{l}\mathrm{R}=300 \Omega, \mathrm{L}=3.2 \mathrm{H}, \\
252 \mathrm{VAR}-120 \mathrm{~V}, 60 \mathrm{~Hz}, \text { precisión de } \pm 5 \%, \mathrm{Q}=10 .\end{array}$ \\
\hline Circuito RC & $\begin{array}{l}\mathrm{R}=1.200 \Omega, \mathrm{C}=2.2 \mu \mathrm{F}, 60 \mathrm{~Hz}, \\
252 \mathrm{VAR}-120 \mathrm{~V}(240 \mathrm{~V} \text { Máx }) \text {, precisión de } \pm 5 \% .\end{array}$ \\
\hline $\begin{array}{l}\text { Motor jaula de } \\
\text { ardilla }\end{array}$ & $175 \mathrm{~W}, 1670 \mathrm{r} / \mathrm{min}, 2018 \mathrm{~V}-1.2 \mathrm{~A}, 3 \phi, 60 \mathrm{~Hz}$. \\
\hline $\begin{array}{l}\text { Transformado } \\
\mathrm{r} 120 \mathrm{~V} / 120 \\
\mathrm{~V}\end{array}$ & $60 \mathrm{VA}, 120 \mathrm{~V} / 208 \mathrm{~V} / 120 \mathrm{~V}$, \\
\hline $\begin{array}{l}\text { Transformado } \\
\mathrm{r} \text { reductor } \\
120 \mathrm{~V} / 60 \mathrm{~V}\end{array}$ & $60 \mathrm{VA}, 120 \mathrm{~V} / 208 \mathrm{~V} / 120 \mathrm{~V}$, \\
\hline $\begin{array}{l}\text { Transformado } \\
\text { r elevador } \\
120 \mathrm{~V} / 180 \mathrm{~V}\end{array}$ & $60.5 \mathrm{AA} / 0.3 \mathrm{~A} / 0.5 \mathrm{~A}, 60 \mathrm{~Hz}$ y $1 \phi$. \\
\hline
\end{tabular}

Aplicando el procedimiento propuesto al motor rotor jaula de ardilla en delta, se tiene como esquema de conexión el indicado en la Fig. 4, además de las curvas de corriente y voltaje obtenidas mostradas en la Fig.5, donde la curva más oscura pertenece a la corriente, que para este caso tiene un valor RMS de 1.14 A y la curva más clara pertenece al voltaje, con su respectivo valor RMS de $110.3 \mathrm{~V}$, también se resalta la importancia de obtener las señales en un ambiente controlado para obtener resultados más reales y eficaces.

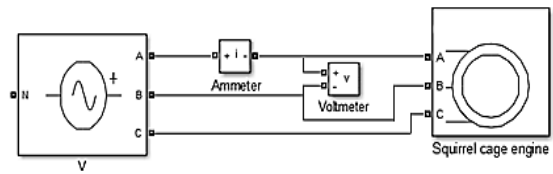

Fig. 4. Esquema de conexión para un motor jaula de ardilla en delta.

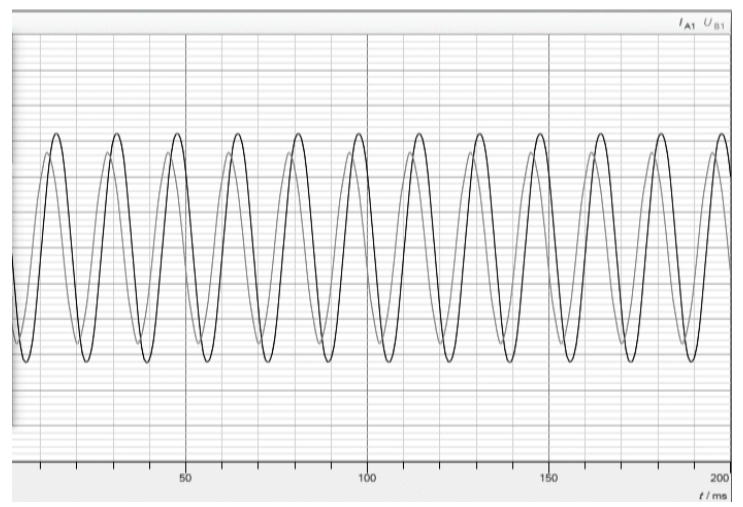

Fig. 5. Curvas de corriente (línea oscura) y voltaje (línea clara) del motor jaula de ardilla en conexión delta.
Las funciones utilizadas para el tratamiento de las señales con el fin de facilitar la obtención de algunos de los parámetros en el entorno Matlab se indican en la tabla IX, donde se utiliza la función load para cargar los 10.000 datos dados por el sensor, la función thd toma la señal y arroja resultados en $\mathrm{dB}$ de los armónicos presentes, es por esto que se utiliza la función db2mag para determinar el valor en magnitud, la función rms, para facilitar el cálculo del valor eficaz de las curvas, la función findpeaks que calcula los máximos picos de la señal cada $0,016 \mathrm{~s}$ que equivalen a $60 \mathrm{~Hz}$, y por último la función xlswrite encargada de guardar los datos en un archivo EXCEL para su tratamiento, ahora bien, se analizaron las curvas de corriente y voltaje correspondientes al motor jaula de ardilla y los parámetros que se obtuvieron al aplicar las ecuaciones de la tabla I se indican en la tabla X.

TABLA IX

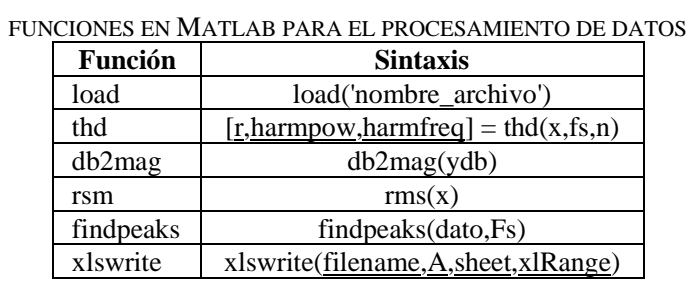

TABLA X

PARÁMETROS DEL MOTOR JAULA DE ARDILL
\begin{tabular}{|l|c|}
\hline \multicolumn{1}{|c|}{ Dato } & Valor \\
\hline FP & 0,6000 \\
\hline THD_I & 0,0116 \\
\hline RMS_I & 1,1455 \\
\hline FACTOR_CRESTA_I & 1,4024 \\
\hline ARM_3_I & 0,0050 \\
\hline ARM_5_I & 0,0118 \\
\hline ARM_7_I & 0,0010 \\
\hline
\end{tabular}

TABLA X (CONTINUACIÓN)

\begin{tabular}{|l|c|}
\hline THD_V & 0,0184 \\
\hline FACTOR_CRESTA_V & 1,4473 \\
\hline ARM_3_V & 0,3930 \\
\hline ARM_5_V & 1,7639 \\
\hline ARM_7_V & 0,8221 \\
\hline
\end{tabular}

El siguiente paso es identificar la carga, estableciendo los parámetros que se encuentran dentro de los rangos (tabla XI), definidos para el motor jaula de ardilla en específico, esto se hace por medio de un código de identificación, en la Fig. 6 la línea punteada con el marcador en forma de rombo indica el límite inferior (LIC), la línea punteada con marcador en forma de triángulo pertenece al límite superior (LSC) y la línea solida con marcador en forma de cuadrado indica el valor de los parámetros correspondientes al motor rotor jaula de ardilla (VALOR), se puede observar que efectivamente la línea correspondiente al valor de los parámetros está dentro de los rangos definidos para esta carga, según este comportamiento se puede observar que efectivamente la carga conectada es un motor jaula de ardilla. 
TABLA XI

RANGOS ESPECÍFICOS DEL MOTOR JAULA DE ARDILLA

\begin{tabular}{|l|l|l|}
\hline \multicolumn{2}{|c|}{ MOTOR JAULA DE ARDILLA DELTA } \\
\hline Parámetro & \multicolumn{1}{|c|}{ LIC } & \multicolumn{1}{c|}{ LSC } \\
\hline FP & 0,6 & 0,6 \\
\hline THD_I & 0,01 & 0,0154 \\
\hline RMS_I & 1,1421 & 1,166 \\
\hline FC_I & 1,395 & 1,4127 \\
\hline ARM_3_I & 0,003 & 0,0081 \\
\hline ARM_5_I & 0,0081 & 0,0164 \\
\hline ARM_7_I & $-0,0001$ & 0,0035 \\
\hline THD_V & 0,0166 & 0,0218 \\
\hline FC_V & 1,4345 & 1,4567 \\
\hline ARM_3_V & 0,1607 & 0,89 \\
\hline ARM_5_V & 1,1554 & 2,1926 \\
\hline ARM_7_V & 0,6618 & 1,2679 \\
\hline
\end{tabular}

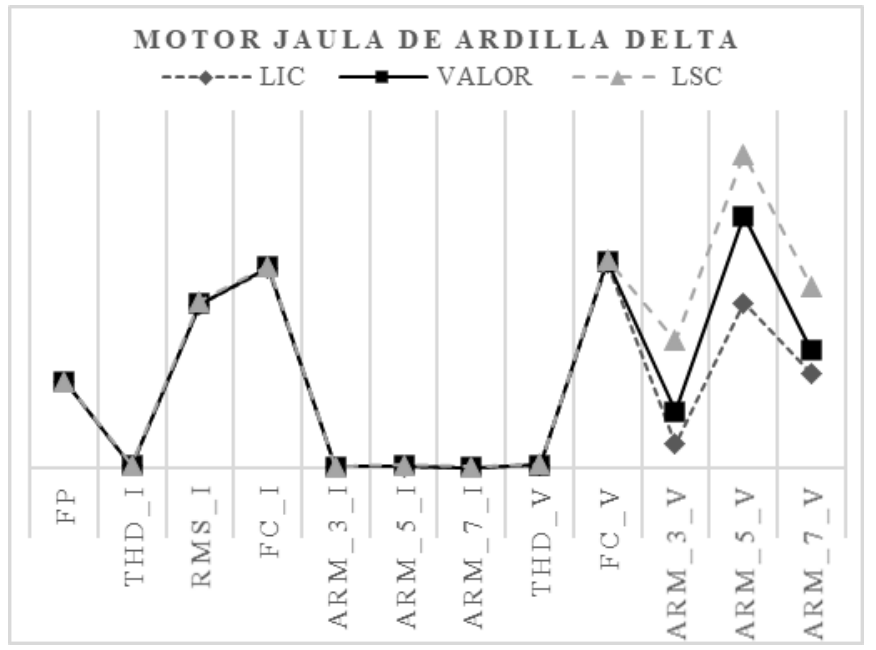

Fig. 6. Resultado grafico de los rangos y parámetros de un motor jaula de ardilla.

Se desarrolló el mismo procedimiento para las cargas mencionadas en la tabla V, Circuito RLC, Circuito RL, Circuito RC, transformador $120 \mathrm{~V} / 120 \mathrm{~V}$, transformador elevador $120 \mathrm{~V} / 180 \mathrm{~V}$, transformador reductor $120 \mathrm{~V} / 60 \mathrm{~V}$, los resultados de estas pruebas fueron satisfactorios, ya que se comprobó que efectivamente el procedimiento propuesto es el indicado para la identificación de las cargas, a continuación como ejemplo, se indica gráficamente los resultados obtenidos para un Circuito RLC (Fig. 7), donde la línea punteada con el marcador en forma de rombo indica el límite inferior (LIC), la línea punteada con marcador en forma de triángulo pertenece al límite superior (LSC) y la línea solida con marcador en forma de cuadrado indica el valor de los parámetros correspondientes al circuito RLC en delta (VALOR), gráficamente se puede observar que uno de los valores esta fuera de los rangos (ARM_5_V), esto pudo ser ocasionado por algún cambio en la red trifásica a la hora de tomar las curvas, paro esto no influye a la hora de identificar la carga.

Por último, para el proceso de validación, se toma como caso de estudio el motor jaula de ardilla con una carga conectada, dicha carga será un electrodinamómetro con las características presentadas en la tabla XII

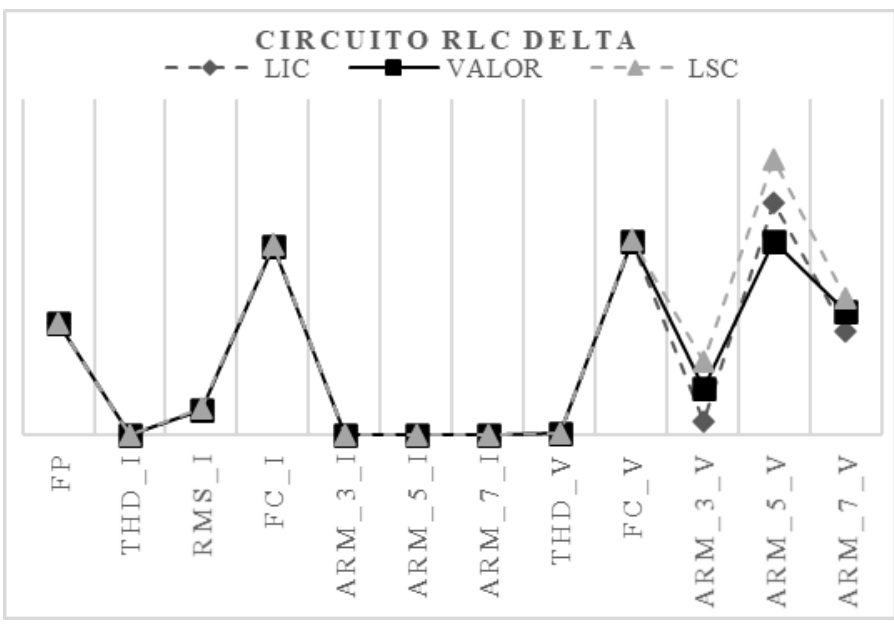

Fig. 7. Resultado grafico de los rangos y parámetros de circuito RLC en delta.

TABLA XII

CARACTERÍSTICAS DE UN ELECTRODINAMÓMETRO.

\begin{tabular}{|c|c|}
\hline \multicolumn{2}{|c|}{ Características } \\
\hline Velocidad & $0-5000 \mathrm{r} / \mathrm{min}$ \\
\hline Par motor & $0-27 \mathrm{LBF}^{*} \mathrm{PLG}$ \\
\hline
\end{tabular}

Para este caso en particular, se le aplicó al electrodinamómetro un voltaje DC de $24 \mathrm{~V}$, esto ejerce un par motor de 1.2 LBR - PLG, las curvas características de voltaje y corriente para el motor jaula de ardilla con carga se muestran en la Fig. 8, donde la curva más clara pertenece a la corriente, que para este caso tiene un valor RMS de $1.20 \mathrm{~A}$ y la curva más oscura pertenece al voltaje, con su respectivo valor RMS de $109.9 \mathrm{~V}$, además se indican los parámetros obtenidos al analizar las curvas correspondientes en la tabla XIII.

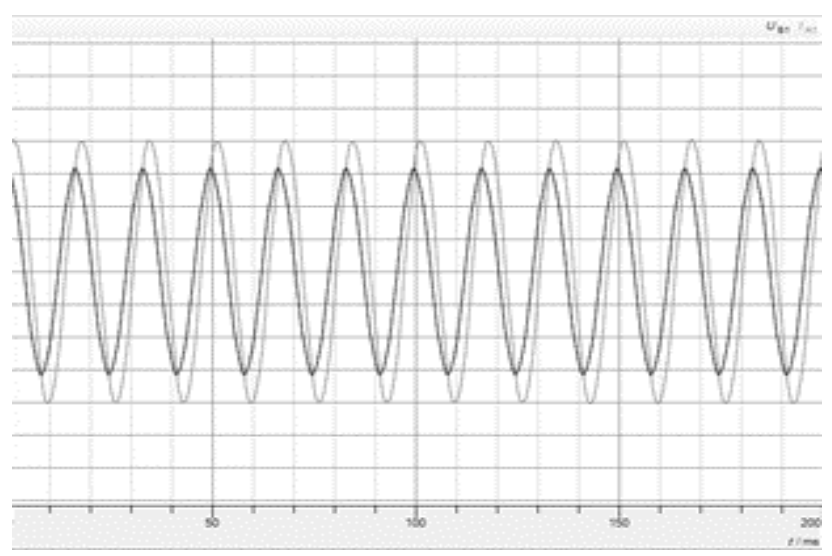

Fig. 8. curvas de corriente (línea clara) y voltaje (línea oscura) del motor jaula de ardilla con carga en conexión delta.

Varios de los parámetros de la tabla XIII no se encuentran entre los rangos definidos para un motor rotor jaula de ardilla con conexión delta indicados en la tabla XI, esto también se puede observar gráficamente, como se indica en la Fig. 9. 
TABLA XIII

PARÁMETROS DEL MOTOR JAULA DE ARDILLA CON CARGA

\begin{tabular}{|l|c|}
\hline \multicolumn{1}{|c|}{ Parámetro } & Valor \\
\hline FP & 0,7800 \\
\hline THD_I & 0,0238 \\
\hline RMS_I & 1,2005 \\
\hline FACTOR_CRESTA_I & 1,4182 \\
\hline ARM_3_I & 0,0024 \\
\hline ARM_5_I & 0,0243 \\
\hline ARM_7_I & 0,0123 \\
\hline THD_V & 0,0162 \\
\hline FACTOR_CRESTA_V & 1,4536 \\
\hline ARM_3_V & 0,5485 \\
\hline ARM_5_V & 1,1649 \\
\hline ARM_7_V & 1,0700 \\
\hline
\end{tabular}

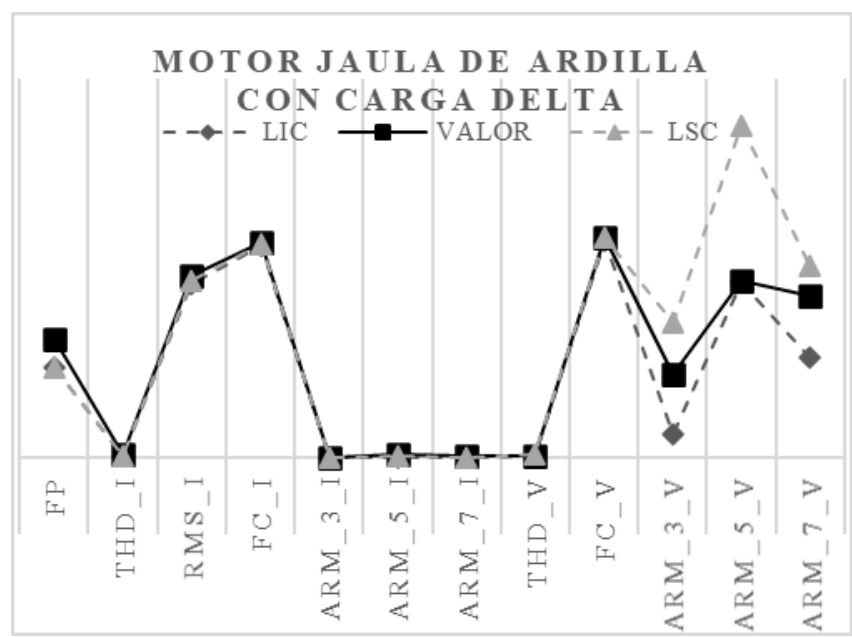

Fig. 9. Resultado grafico de los rangos de un motor jaula de ardilla y parámetros de un motor jaula de ardilla con carga.

Es por lo que como se indica en la tabla XIV se definen nuevos rangos específicos para esta nueva carga, agregado a los nuevos parámetros el error muestral definido anteriormente para un motor jaula de ardilla.

TABLA XIV

CRITERIOS PERFIL DE CARGA PARA MOTOR JAULA DE ARDILLA CON CARGA

\begin{tabular}{|l|c|l|c|c|c|}
\hline Parámetro & Valor & $\begin{array}{c}\text { Desviación } \\
\text { estándar }\end{array}$ & $\begin{array}{c}\text { Error } \\
\text { muestral }\end{array}$ & LSC & LIC \\
\hline FP & 0,7800 & $1,11022 \mathrm{E}-16$ & $9,05795 \mathrm{E}-17$ & 0,7800 & 0,7800 \\
\hline THD_I & 0,0238 & 0,001164322 & 0,000949933 & 0,0248 & 0,0229 \\
\hline RMS_I & 1,2005 & 0,004721052 & 0,003851754 & 1,2043 & 1,1966 \\
\hline F_C_I & 1,4182 & 0,003426865 & 0,002795868 & 1,4210 & 1,4154 \\
\hline ARM_3_I & 0,0024 & 0,001182097 & 0,000964435 & 0,0033 & 0,0014 \\
\hline ARM_5_I & 0,0243 & 0,001620376 & 0,001322012 & 0,0256 & 0,0229 \\
\hline ARM_7_I & 0,0123 & 0,000791834 & 0,000646032 & 0,0130 & 0,0117 \\
\hline THD_V & 0,0162 & 0,000962384 & 0,000785178 & 0,0170 & 0,0155 \\
\hline F_C_V & 1,4536 & 0,004169408 & 0,003401685 & 1,4570 & 1,4502 \\
\hline ARM_3_V & 0,5485 & 0,165099605 & 0,134699424 & 0,6832 & 0,4138 \\
\hline ARM_5_V & 1,1649 & 0,19681925 & 0,160578457 & 1,3255 & 1,0043 \\
\hline ARM_7_V & 1,0700 & 0,127925878 & 0,104370583 & 1,1744 & 0,9656 \\
\hline
\end{tabular}

A continuación, se muestra el resultado de las curvas características de corriente y voltaje de un motor jaula de ardilla con carga Fig. 10, donde la curva más clara pertenece a la corriente, que para este caso tiene un valor RMS de $1.20 \mathrm{~A}$ y la curva más oscura pertenece al voltaje, con su respectivo valor RMS de $110.2 \mathrm{~V}$, tomado en otro momento, es así, como se obtienen de nuevo los parámetros indicados en la tabla XV

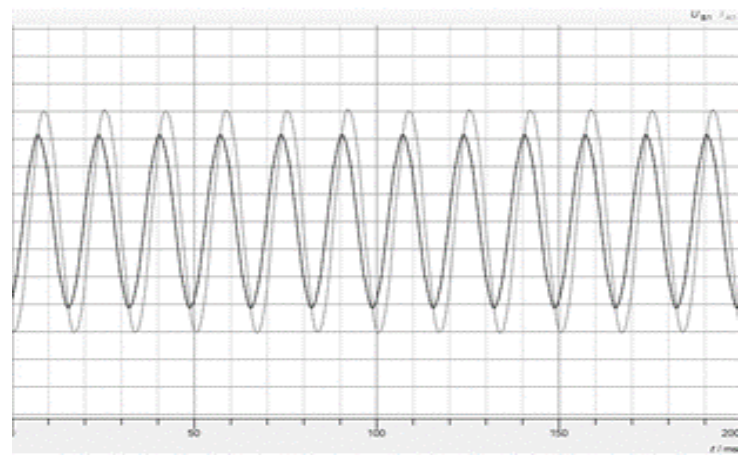

Fig. 10. curvas de corriente (línea clara) y voltaje (línea oscura) del motor jaula de ardilla con carga en conexión delta.

TABLA XV

PARÁMETROS OBTENIDOS DEL ANÁLISIS DEL MOTOR JAULA DE ARDILLA CON CARGA

\begin{tabular}{|c|c|}
\hline Parámetro & Valor \\
\hline FP & 0,7800 \\
\hline THD_I & 0,0229 \\
\hline RMS_I & 1,2099 \\
\hline FACTOR_CRESTA_I & 1,4000 \\
\hline ARM_3_I & 0,0057 \\
\hline ARM_5_I & 0,0236 \\
\hline ARM_7_I & 0,0121 \\
\hline THD_V & 0,0160 \\
\hline FACTOR_CRESTA_V & 1,4487 \\
\hline ARM_3_V & 0,3009 \\
\hline ARM_5_V & 1,1527 \\
\hline ARM_7_V & 1,0825 \\
\hline
\end{tabular}

Como se observa gráficamente en la Fig. 11, los valores de los parámetros están dentro de los rangos definidos, para el motor jaula de ardilla con carga.

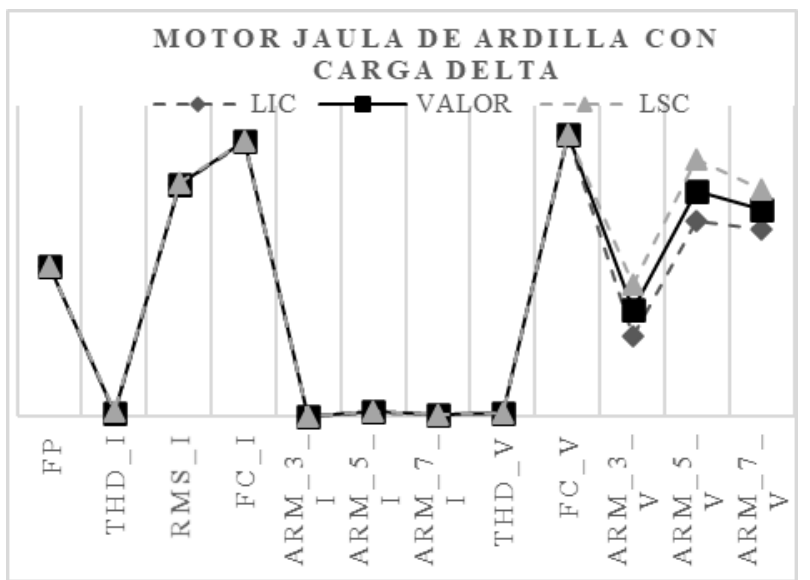

Fig. 11. Resultado grafico de los rangos y parámetros de un motor jaula de ardilla con carga.

Es así como se define una nueva plantilla que corresponde a un motor jaula de ardilla con carga, como se indica en la tabla XVI 
TABLA XVI

PLANTILLA DE IDENTIFICACIÓN PARA EL MOTOR JAULA DE ARDILLA CON CARGA

\begin{tabular}{|l|c|c|c|}
\hline \multicolumn{4}{|c|}{ Motor jaula de ardilla con carga (Electrodinamómetro) } \\
\hline CONEXIÓN: & Delta \\
\hline ID: & MJA_C_101 \\
\hline \multicolumn{2}{|l|}{ Parámetro } & $\begin{array}{c}\text { Rango } \\
\text { inferior }\end{array}$ & $\begin{array}{c}\text { Rango } \\
\text { superior }\end{array}$ \\
\hline FP & $7,578 \%$ & 0,7800 & 0,7800 \\
\hline THD_I & $0,164 \%$ & 0,0248 & 0,0229 \\
\hline RMS_I & $14,568 \%$ & 1,2043 & 1,1966 \\
\hline FACTOR_CRESTA_I & $17,717 \%$ & 1,4210 & 1,4154 \\
\hline ARM_3_I & $0,071 \%$ & 0,0033 & 0,0014 \\
\hline ARM_5_I & $0,169 \%$ & 0,0256 & 0,0229 \\
\hline ARM_7_I & $0,019 \%$ & 0,0130 & 0,0117 \\
\hline THD_V & $0,249 \%$ & 0,0170 & 0,0155 \\
\hline FACTOR_CRESTA_V & $18,291 \%$ & 1,4570 & 1,4502 \\
\hline ARM_3_V & $6,251 \%$ & 0,6832 & 0,4138 \\
\hline ARM_5_V & $22,950 \%$ & 1,3255 & 1,0043 \\
\hline ARM_7_V & $11,972 \%$ & 1,1744 & 0,9656 \\
\hline
\end{tabular}

En este artículo se exponen criterios de evaluación del perfil de carga de acuerdo con el comportamiento, que permitieron la identificación de las mismas. Para ello se analizaron las formas de onda de voltaje y corriente calculando parámetros de identificación con los cuales se determinan rangos de pertenecía para asociar el perfil de carga a los criterios de evaluación. Dichos rangos son mostrados en la Tabla V, los cuales fueron validados mediante el procedimiento propuesto y el resultado permite la identificación de las cargas en estudio.

De igual forma, se comprobó la importancia del comportamiento de la red eléctrica en la cual se realizaron las mediciones, ya que la presencia de eventos transitorios de tensión fueron causa potencial de perturbaciones, lo que afectó no solo la calidad de datos de la forma de onda en cada registro, sino también en los parámetros que se van a calcular presentando alteraciones en sus valores, por ende se determina hacer las pruebas en horarios de baja demanda en un ambiente controlado, teniendo sólo la conexión de las cargas conocidas para obtener resultados más exactos.

También, se verificó la importancia de determinar las características eléctricas de la red como de las cargas en estudio, además, determinar la frecuencia de muestreo comprobando con el estudio realizado que para frecuencias de $60 \mathrm{~Hz}$ es necesario tomar 12 ciclos de la señal para análisis de formas de ondas, de igual forma, para realizar mediciones de alta precisión se debe usar un equipo que soporte dichas características sin transformaciones en las formas de onda de voltaje y corriente medidas para aplicar correctamente el procedimiento.

Se establecen herramientas analíticas de datos para verificar el comportamiento de los valores obtenidos de las formas de onda de voltaje y corriente, resaltando la importancia de tener un grupo considerable de datos, ya que para analizar la varianza y dispersión de los mismos es importante tener más de una muestra de las señales medidas.
Por último, se realizó la verificación del procedimiento y de los criterios de evaluación, mediante una validación propuesta que permitió conectar cargas nuevas y conocidas para ser identificadas, de esto se concluye, que el procedimiento es certero, es decir, que al medir las cargas ya definidas, el procedimiento es capaz de verificar que tipo de carga está conectada. Las nuevas cargas se consideran aplicando pequeñas variaciones en las características de los circuitos eléctricos ya conectados, es necesario tomar una medición y sacar los valores de los parámetros teniendo en cuenta el error muestral ya obtenido, con lo que se pretende identificar la nueva carga.

\section{REFERENCIAS}

[1] H. K. Siu \& H. W. Ngan, Automatic power quality recognition system using wavelet analysis, 2004 IEEE International Conference on Electric Utility Deregulation, Restructuring and Power Technologies. Proceedings, Hong Kong, China, 2004, pp. 311-316 Vol.1, doi: 10.1109/DRPT.2004.1338513.

[2] Meléndez J., Herraiz, S., Colomer, J., Sánchez, J. \& Castro, M. Monitorización y diagnóstico de la calidad de onda. 2006. Automática e instrumentación, n. ${ }^{\circ} 371$.

[3] A. M. Castro \& E. Ramos. Efectos de los armónicos en los motores de inducción (tesis de pregrado). (2008). Universidad de la Salle, Bogotá D.C., Colombia.

[4] Valencia J. A., García C. A. \& Martínez W. A. Proyección de Demanda de Energía Eléctrica y potencia máxima en Colombia 2016. Unidad de Planeación Minero-Energética-UPME. pp. 1-32.

[5] Ministerio de Minas y Energía MME. Plan de acción indicativo de eficiencia energética 2017- 2022. Unidad de Planeación MineroEnergética-UPME, República de Colombia.

[6] IEEE Recommended Practice for Monitoring Electric Power Quality, in IEEE Std 1159-2009 (Revision of IEEE Std 1159-1995), pp.1-94, 26 June 2009, doi: 10.1109/IEEESTD.2009.5154067.

[7] K. Umeh and A. Mohamed, Intelligent system for identification of harmonics originating from single phase nonlinear loads, Proceedings. IEEE SoutheastCon, 2005., Ft. Lauderdale, FL, USA, 2005, pp. $137-$ 142, doi: 10.1109/SECON.2005.1423233.

[8] S. F. Legowski, A. H. M. Sadrul Ula and A. M. Trzynadlowski, Instantaneous stator power as a medium for the signature analysis of induction motors, IAS '95. Conference Record of the 1995 IEEE Industry Applications Conference Thirtieth IAS Annual Meeting, Orlando, FL, USA, 1995, pp. 619-624 vol.1, doi: 10.1109/IAS.1995.530356.

[9] G. Frigo, G. Giorgi, M. Bertocco and C. Narduzzi, Multifunction phasor analysis for distribution networks, 2016 IEEE International Workshop on Applied Measurements for Power Systems (AMPS), Aachen, 2016, pp. 1-6, doi: 10.1109/AMPS.2016.7602868.

[10] D. Belega, D. Macii and D. Petri, Power system frequency estimation accuracy of improved DFT-based algorithms over short intervals, 2016 IEEE International Workshop on Applied Measurements for Power Systems (AMPS), Aachen, 2016, pp. 1-6, doi: 10.1109/AMPS.2016.7602810.

[11] J. A. de la O Serna, Dynamic Phasor Estimates for Power System Oscillations, in IEEE Transactions on Instrumentation and Measurement, vol. 56, no. 5, pp. 1648-1657, Oct. 2007, doi: 10.1109/TIM.2007.904546.

[12] R.K. Mai, Z. He, L. Fu, W. He \& Z.Q. Bo. Dynamic phasor and frequency estimator for phasor measurement units. 2010. Generation, Transmission \& Distribution, IET. 4. 73 - 83, doi: 10.1049/ietgtd.2009.0320.

[13] D. Petri, D. Fontanelli and D. Macii, A Frequency-Domain Algorithm for Dynamic Synchrophasor and Frequency Estimation, in IEEE Transactions on Instrumentation and Measurement, vol. 63, no. 10, pp. 2330-2340, octubre 2014, doi: 10.1109/TIM.2014.2308996.

[14] P. Castello, C. Muscas and P. A. Pegoraro, Performance comparison of algorithms for synchrophasors measurements under dynamic conditions, IEEE International Workshop on Applied Measurements 
for Power Systems (AMPS), Aachen, septiembre 2011, pp. 25-30, doi: 10.1109/AMPS.2011.6090351.

[15] G. Barchi, D. Macii and D. Petri, Synchrophasor Estimators Accuracy: A Comparative Analysis, in IEEE Transactions on Instrumentation and Measurement, vol. 62, no. 5, pp. 963-973, mayo 2013, doi: 10.1109/TIM.2012.2236776.

[16] M. A. Platas-Garza and J. A. de la O Serna, Polynomial Implementation of the Taylor-Fourier Transform for Harmonic Analysis, in IEEE Transactions on Instrumentation and Measurement, vol. 63, no. 12, pp. 2846-2854, diciembre 2014, doi: 10.1109/TIM.2014.2324191.

[17] M. D. Kušljević and J. J. Tomić, Multiple-Resonator-Based Power System Taylor-Fourier Harmonic Analysis, in IEEE Transactions on Instrumentation and Measurement, vol. 64, no. 2, pp. 554-563, febrero 2015, doi: 10.1109/TIM.2014.234559.

[18] Weiqiang Chen, A. Ulatowski and A. M. Bazzi, Threshold-based power grid fault diagnosis, IEEE Power \& Energy Society General Meeting, Denver, CO, octubre 2015, pp. 1-5, doi: 10.1109/PESGM.2015.7286503.

[19] M. Sanaullah and M. H. Chowdhury, A new real pole delay model for RLC interconnect using second order approximation, IEEE 57th International Midwest Symposium on Circuits and Systems (MWSCAS), College Station, TX, Agosto 2014, pp. 238-241, doi: 10.1109/MWSCAS.2014.6908396.

[20] S. Hongwei, L. Yuli and C. Guangfeng, Relations between the Standard Variance and the Allan Variance, International Conference on Computational and Information Sciences, Chengdu, diciembre 2010, pp. 66-67, doi: 10.1109/ICCIS.2010.23.

[21] S. Zbigniew, Power system properties periodical time variance investigations: hardware and software tools development, IEEE Instrumentation \& Measurement Technology Conference IMTC, Warsaw, mayo 2007, pp. 1-5, and doi: 10.1109/IMTC.2007.379203.

[22] Allah, R. Correlation-based synchro-check relay for power systems. IET Generation, Transmission \& Distribution, vol. 12, no. 5, marzo 2018, pp.1109-1120, doi: 10.1049/iet-gtd.2017.0448.

[23] Saleh, S., EL-Hoshy, S. and Gouda, O. Proposed diagnostic methodology using the crosscorrelation coefficient factor technique for power transformer fault identification. IET Electric Power Applications, vol. 11, no. 3, marzo 2017, pp.412-422, doi: 10.1049/ietepa.2016.0545

[24] Zhou, N., Lou, X., Yu, D., Wang, Q. and Wang, J. Harmonic InjectionBased Power Fluctuation Control of Three-Phase PV Systems under Unbalanced Grid Voltage Conditions. IEEE Energies, vol. 8, no. 2, Enero 2015, pp.1390-1405, doi: 10.3390/en8021390.

Francisco Franco Obando Díaz es Ingeniero Físico y Magister en Electrónica y Telecomunicaciones de la Universidad del Cauca (Colombia) Docente investigador desde 2007, adscrito al departamento de Electrónica, Instrumentación y Control de la Universidad del Cauca, apoyo al grupo de investigación AI con código ORCID https://orcid.org/0000-0001-5666-6969.

Paula Andrea Bolaños Vega es Ingeniera en Automática Industrial, con énfasis en sistemas de potencia, coordinadora del semillero de investigación "Gestión de la Energía" de la Universidad del Cauca, con código ORCID https://orcid.org/0000-0001-9834-1206.

Gisselle Alejandra Palomino Dorado es Ingeniera en Automática Industrial de la Universidad del Cauca, con énfasis en sistemas de potencia, coordinadora del semillero de investigación "Gestión de la Energía" de la misma institución, con código ORCID https://orcid.org/0000-0001-66217251. 